\title{
Atenção à saúde da mulher durante a pandemia COVID-19: orientações para o trabalho na APS
}

\author{
Women's health care during COVID-19 pandemic: a guide for primary health care
}

\section{Atención a la salud de la mujer durante la pandemia de COVID-19: pautas para el trabajo de APS}

\author{
Carolina Lopes de Lima Reigada ${ }^{10}$, Clarice de Azevedo Sarmet Loureiro Smiderle ${ }^{1 \oplus}$ \\ ${ }^{1}$ Universidade do Estado do Rio de Janeiro (UERJ), Rio de Janeiro, RJ, Brasil \\ ${ }^{2}$ Secretária Municipal De Saúde Do Rio De Janeiro (Sms-Rj), Rio De Janeiro, RJ, Brasil
}

\section{Resumo}

A pandemia pelo novo coronavírus causou uma crise sanitária mundial, com repercussões mais graves em sistemas de saúde já sobrecarregados e em sociedades mais desiguais. Neste artigo, revisou-se a literatura sobre pontos importantes no atendimento à saúde da mulher durante pandemias nos serviços de Atenção Primária à Saúde (APS). Destaca-se o papel crucial da APS na prevenção de iniquidades. No tocante à saúde da mulher, é importante o foco na atuação contra a violência doméstica, manutenção de atenção ao ciclo gravídico-puerperal, contracepção e condições potencialmente graves, como Infecções Sexualmente Transmissíveis e seguimento oncológico.

Palavras-chave: Assistência Integral à Saúde; Cobertura Universal do Seguro de Saúde;Violência contra a Mulher; Atenção Primária à Saúde; Infecções por Coronavirus

\begin{abstract}
The new coronavirus pandemic caused a global health crisis, with more serious repercussions on already overburdened health systems and more unequal societies. In this article, the literature about important points in women's health care during pandemics in Primary Health Care (PHC) services was reviewed. PHC's crucial role in preventing inequities is highlighted. With regard to women's health, it is important to focus on actions against domestic violence, maintaining attention to the pregnancy-puerperal cycle, contraception and potentially serious conditions, such as Sexually Transmitted Infections and oncological follow-up.
\end{abstract}

Keywords: Comprehensive Health Care; Universal Health Insurance; Violence Against Women; Primary Health Care; Coronavirus Infections

\section{Resumen}

La nueva pandemia de coronavirus causó una crisis de salud global, con repercusiones más serias en los sistemas de salud ya sobrecargados y en sociedades más desiguales. En este artículo, revisamos la literatura sobre puntos importantes en el cuidado de la salud de las mujeres durante las pandemias en los servicios de Atención Primaria de Salud (APS). Se destaca el papel crucial de APS en la prevención de las inequidades. Con respecto a la salud de las mujeres, es importante centrarse en la acción contra la violencia doméstica, manteniendo la atención al ciclo embarazopuerperal, la anticoncepción y las condiciones potencialmente graves, como las infecciones de transmisión sexual y el seguimiento oncológico.

Palabras clave: Atención Integral de Salud; Cobertura Universal del Seguro de Salud; Violencia contra la Mujer, Atención Primaria de Salud; Infecciones por Coronavirus

Como citar: Reigada CLL, Smiderle CASL. Atenção à saúde da mulher durante a pandemia COVID-19: orientações para o trabalho na APS. Rev Bras Med Fam Comunidade. 2021;16(43):2535. https://doi.org/10.5712/rbmfc16(43)2535 
A disseminação do novo coronavírus mostrou como, em diferentes territórios, vive-se uma epidemia de iniquidade. Por este ângulo, pode-se dizer que essa pandemia tem cor, classe e gênero - além dos fatores de risco biológicos. De fato, crises geram maior impacto nos segmentos populacionais mais frágeis, expondo desigualdades existentes na tessitura social ${ }^{1,2,3}$.

O distanciamento social é a medida adotada pela maioria dos países na tentativa de diminuir a velocidade de contágio. No Brasil, cada estado vem se organizando de acordo com sua realidade epidemiológica e estrutural, mas serviços essenciais, como os de saúde, permanecem abertos - apesar de diminuição importante de sua frequentação ${ }^{4,5,6}$.

Diversas entidades chamaram atenção para o aumento da violência doméstica contra mulheres e crianças durante o período de isolamento social $7,8,9,10,11,12$. O convívio prolongado, dificuldades financeiras e interrupção de atividades de trabalho e lazer são possíveis explicações ${ }^{7,9,11,12}$. Ressalta-se, ainda, que as famílias são afetadas de forma heterogênea, de acordo com diferentes condicionantes a que estão submetidas ${ }^{9,10,12}$.

A pandemia também representa uma barreira de acesso a serviços de saúde, já que estes estão reorganizados para atendimento prioritário aos pacientes possivelmente infectados, e com profissionais afastados do atendimento por diversos motivos, com sobrecarga dos remanescentes. Além disso, as pessoas evitam ir aos serviços, pelas recomendações de afastamento social e pelo medo de contaminação ${ }^{10,13,14,15}$.

Assim, a Atenção Primária à Saúde (APS) torna-se crucial para evitar o aprofundamento da lei de cuidados inverso ${ }^{16}$ e da iniquidade, mantendo vigilância sobre famílias e indivíduos mais frágeis. Para estes, o impacto da pandemia é não só direto (com a possibilidade de contágio e adoecimento), mas também indireto, seja pela perda de renda e outras necessidades básicas ou pela falta de acompanhamento de outras condições de saúde, que também ocasionam morbidade e mortalidade importantes ${ }^{10}$.

Para esse documento, foi feita uma busca na literatura sobre pontos importantes da atenção à saúde da mulher durante pandemias para orientar equipes de APS durante a pandemia da COVID-19 a melhor planejar seu processo de trabalho.

\section{Violência contra a mulher}

O medo do novo coronavírus, o afastamento da rede socioafetiva, a ameaça financeira, o aumento do consumo de álcool e drogas e maior convívio domiciliar são possíveis explicações para o aumento da violência doméstica ${ }^{7,8,9,10}$. A proximidade do agressor dificulta o contato telefônico com serviços de ajuda; e a diminuição da mobilidade dificulta o afastamento da situação de violência, também aumentando a sensação de poder e controle do abusador. O maior convívio doméstico aumenta o trabalho invisível e a sobrecarga das mulheres, tornando-as mais fragilizadas e suscetíveis à violência psicológica e coerção7,8,9,10,17,18,19. Esse dado torna-se mais preocupante com o possível desmonte ou enfraquecimento de estruturas de proteção à mulher, tanto comunitárias quanto governamentais $7,8,11$. No mês de abril/2020, enquanto registros criminais diminuíram, as denúncias de violência contra a mulher aumentaram ${ }^{11,17,19}$. Entretanto, com a suspensão das prisões preventivas e audiências, a proteção das mulheres fica ainda mais comprometida ${ }^{11}$.

A APS é estratégica pela proximidade às realidades das famílias e mulheres do território. Os Agentes Comunitários de Saúde (ACS) atuam na promoção, prevenção e controle de agravos e, sendo membros da comunidade, são essenciais na identificação da violência intrafamiliar. Contudo, muitas vezes esses profissionais 
não compõem as equipes de APS, ou se sentem inseguros e despreparados para esta abordagem, com limitação de sua capacidade de reconhecimento e atuação. A parca integração da APS com outros equipamentos sociais também contribui para a baixa resolutividade desses $\operatorname{casos}^{20,21,22,23,24}$. Neste período, torna-se necessário fomento à discussão nas equipes sobre violência intrafamiliar, envolvendo todos os profissionais na detecção dos casos e reflexão sobre formas eficazes de abordagem ${ }^{9,17,18,19}$. Salienta-se que o relato da violência necessita de ambiente receptivo e estimulador para que a mulher possa sentir-se segura e que o profissional esteja apto e atento a perceber essa possível agenda oculta, especialmente durante a pandemia ${ }^{25,26}$.

Há a recomendação de reforço e divulgação na unidade de saúde e na comunidade do uso de serviços de denúncia por telefone ou internet, como Disque 180 e Disque 100 - ambos disponíveis 24 horas por dia, todos os dias da semana, com perspectiva de ampliação de atendimento pelo whatsapp ${ }^{11,27,28,29}$, como foi feito em diferentes países ${ }^{30}$. O Disque 100, serviço para denúncia de violações de direitos humanos, iniciou canal específico para eventos referentes à COVID-19, estendendo o acesso a brasileiras e brasileiros que residem no exterior $^{29}$. Foi lançado o aplicativo Direito Humanos Brasil e os sites ouvidoria.mdh.gov.br, disque100.mdh.gov. br e ligue180.mdh.gov.br para ampliar as oportunidades de denúncias através de celular, com possibilidade de envio de arquivos, pela própria mulher ou por terceiros ${ }^{11,16,19}$. Mesmo assim, sabe-se que os números de violência contra a mulher são maiores que os dos registros oficiais, já que muitas vítimas sentem-se inibidas de denunciar por vergonha, medo, dependência, sentimento de impunidade e proximidade física do agressor. Vários estados lançaram campanhas sobre violência doméstica nas mídias sociais, reforçando o uso desses instrumentos ${ }^{7}$.

Deve-se levar em conta que as equipes de Saúde da Família podem ter registro ou conhecimento de mulheres que vivem situação de violência doméstica, e o contato frequente com elas por telefone, ou pelos ACS, pode facilitar a abordagem inicial. Espalhar cartazes com os números de denúncia e aumentar a discussão sobre o assunto é outra estratégia. Também a proposta de atendimento remoto para abusadores é um recurso, implantado em alguns países, que oferece suporte em situações em que estes entendem estar perdendo o controle ${ }^{31}$. Por fim, a Organização das Nações Unidas (ONU) orienta a ininterrupção de serviços judiciais no processamento dos agressores; evitar a libertação provisória de prisioneiros que foram condenados por violência contra mulher; declarar abrigos e serviços para mulheres em situação de violência como essenciais, de forma que fiquem abertos durante a pandemia ${ }^{8}$; dentre outras possíveis ações ${ }^{7}$.

\section{Apontamentos sobre assistência à saúde da mulher}

Um sistema de saúde resiliente é capaz de responder rapidamente a uma crise na saúde, enquanto mantém serviços essenciais ${ }^{15}$. Nesse sentido, o Ministério da Saúde, através de notas técnicas ${ }^{32,33,34}$, reforça a necessidade de se manter atendimento a mulheres durante gestação e puerpério; assim como a investigação ou tratamento de condições cuja interrupção represente risco para a saúde da mulher, como neoplasias, sangramento uterino aumentado e infecções sexualmente transmissíveis. Considerando-se que há evidências científicas limitadas sobre o impacto da COVID-19 na embriogênese e saúde materno-fetal, torna-se ainda mais importante a contracepção eficaz nesse momento. A infecção não parece alterar a segurança dos métodos contraceptivos, porém houve relatos de possível transmissão vertical, crescimento uterino retardado, maiores taxas de prematuridade (e maior número de cesarianas) e sofrimento fetal ${ }^{35,36,37}$.

Dessa forma, a garantia de acesso à contracepção durante a pandemia é de extrema relevância. O contexto da pandemia pode levar a desabastecimento ou redução do transporte de medicamentos e 
insumos para contracepção, exigindo atenção pelos gestores. Na perspectiva de se reduzir a frequentação dos serviços de saúde sem limitar o acesso ao planejamento reprodutivo, existe a possibilidade de liberação de mais cartelas de contraceptivo oral às mulheres que já fazem uso desse método. Já no caso de mulheres que utilizam preservativos ou métodos naturais de contracepção, pode-se considerar fornecer antecipadamente contracepção de emergência para caso de necessidade. A inserção de DIU, inclusive no pós-parto imediato, é outra forma de se garantir o acesso a um método contraceptivo e evitar idas repetidas a unidades de saúde ${ }^{10,12,13,14,15,32,33,35,38}$.

Deve-se lembrar que o pré-natal de baixo risco realizado na APS é capaz de reduzir a mortalidade perinatal e infantilis9,40,41,42. Além disso, acesso a contracepção eficaz e oportuna (inclusive acesso a aborto seguro) leva à redução de mortalidade materna e gravidez não planejada, aumenta a autonomia da mulher, com impacto na qualidade de vida e renda familiar. Deve-se lembrar que, apesar da disponibilidade gratuita de diversos métodos contraceptivos, o acesso aos mesmos ainda é desigual, com prejuízo entre as mais pobres e com menor tempo de escolaridade ${ }^{10,13,14,15,35}$.

O maior risco de infecções sexualmente transmissíveis durante a pandemia para mulheres em situação de violência doméstica ou em outras condições de risco psicossocial, como o uso de álcool ou drogas que inibem a capacidade de negociar práticas sexuais seguras, pobreza, entre outras situações de vulnerabilidade, também precisa ser reconhecido, devendo ser uma prioridade para serviços de saúde, inclusive de APS ${ }^{43}$.

Os efeitos da pandemia são mais graves em sistemas de saúde já sobrecarregados, como visto durantes epidemias por Ebola no continente africano. Entre 2014-2015, na África Ocidental, houve diminuição na atenção ao pré-natal e parto, contracepção, entre outros serviços, culminando em quatro vezes mais mortes por doenças evitáveis. Apesar da gravidade do Ebola durante gestação e parto, as atividades de planejamento reprodutivo e atenção ao ciclo gravídico-puerperal foram muito impactadas com redução do acesso, tanto pelo medo da população de frequentar os serviços quanto pela falta de profissionais para atendimento, e esse déficit não foi rapidamente revertido após a epidemia ${ }^{13}$. Em Serra Leoa, entre 2013 e 2016, estima-se que uma diminuição de 50\% no acesso causou 2800 mortes por malária, HIVIAIDS e tuberculose - correspondente a $3 / 4$ das mortes diretas por Ebola ${ }^{15}$. Além disso, a diminuição da atenção à saúde de gestantes, parturientes e puérperas levou ao acréscimo de 3600 mortes maternas, neonatais e de natimortos, um número próximo da quantidade de mortes causadas pela própria epidemia ${ }^{15}$. A frequentação de serviços de planejamento familiar também reduziu drasticamente, em diferentes países. Acontecendo o mesmo na pandemia de COVID-19, as consequências podem envolver aumento do número de gestações indesejadas e abortos inseguros, além de mortes maternas e neonatais e, ainda, impacto em infecções sexualmente transmissíveis. Os efeitos podem se prolongar durante a fase de recuperação da pandemia, atingindo novamente grupos desfavorecidos e negligenciados, revertendo ganhos alcançados nas últimas décadas ${ }^{10,13,14,15,35}$.

\section{Conclusão}

Epidemias agravam as já existentes desigualdades vivenciadas por meninas e mulheres, especialmente se estão em situações de vulnerabilidade. Sendo a APS o principal ponto de acesso da população ao sistema de saúde, é fundamental o empenho para que a sua resposta à pandemia não reproduza ou sustente desigualdades ${ }^{9,10}$. 
Acesso adequado aos serviços de atenção ao ciclo gravídico-puerperal, planejamento reprodutivo, acolhimento em situações de violência e outras situações que tragam ameaça à saúde da mulher devem ser considerados essenciais e mantidos ${ }^{8,9,10}$. Os meios de abordagem, realização de consultas e acompanhamentos relevantes podem incluir contato remoto (por telefone e meios digitais) ou presencial, na unidade de saúde ou por meio de visita domiciliar, dando-se a necessária atenção a medidas recomendadas para proteção, como uso de máscaras, higiene das mãos, distanciamento físico, desinfecção de superfícies e utensílios, uso de equipamentos de proteção individual, isolamento de casos suspeitos ou confirmados, permissão de acompanhantes apenas se necessário e comunicação efetiva com os usuários ${ }^{32,33,34,44,45}$. Serviços que dispõem de profissionais do Núcleo de Apoio à Saúde da Família (NASF) devem adequar suas atividades para potencialização de suas funções, visando à integralidade nos atendimentos a pessoas e famílias, mesmo durante a pandemia ${ }^{44}$.

O compartilhamento de informações claras, consistentes e atualizadas sobre prevenção de transmissão e sinais de gravidade são medidas de promoção de saúde. Como para outras ações, o contato com a população por meios de comunicação (como telefone, vídeos curtos e imagens instrutivas) deve ser estimulado, envolvendo demais equipamentos do território, com articulação junto a redes de apoio comunitárias e de ações sociais ${ }^{7,8,9,44}$.

Existem poucos estudos de alta qualidade no que se refere ao processo de trabalho na APS durante pandemias. Porém, é fundamental considerar os dados disponíveis, as experiências anteriores e aspectos individuais e coletivos na assistência à saúde. É fundamental ultrapassar práticas em saúde que ainda se restringem a soluções técnicas, abarcando as dinâmicas de vida, lida, desafios, recursos da população, através da acessibilidade e do diálogo. É, portanto, essencial que se amplie o que Vasconcelos e Vasconcelos (2019) chamam de "dimensões de solidariedade, amorosidade e autonomia entre as pessoas", a começar pela relação entre profissionais da atenção primária à saúde e mulheres pelas quais as equipes devem se corresponsabilizar ${ }^{46}$.

A pandemia atual por COVID-19 provavelmente não deverá ser a última durante o Antropoceno, à medida que cada vez mais ambientes e biomas são destruídos pela ação humana. Esperamos que esse texto ajude profissionais da APS a tomar as melhores decisões sobre seu processo de trabalho nesses tempos tão desafiadores.

\section{AGRADECIMENTOS}

Agradecemos a MNM que participou da aquisição de dados e aprovação da versão final.

\section{Contribuição dos autores}

CLLR - participou da concepção do trabalho, aquisição e interpretação dos dados, elaboração do texto, aprovação da versão final.

CASLS - participou da concepção do trabalho, aquisição e interpretação dos dados, elaboração do texto, aprovação da versão final.

\section{Conflitos de interesse}

Os autores declaram não haver conflitos de interesse. 


\section{Referências Bibliográficas}

1. Kirby T. Evidence mounts on the disproportionate effect of COVID-19 on ethnic minorities. Lancet Respir Med 2020 Published Online May 8, 2020. doi: http://doi.org/10.1016/S2213-2600(20)30228-9 DOI: https://doi.org/10.1016/S2213-2600(20)30228-9

2. Williams DR, Cooper LA. COVID-19 and Health Equity-A New Kind of "Herd Immunity". JAMA. Published online May 11, 2020. doi: http://doi.org/10.1001/jama.2020.8051 DOI: https://doi.org/10.1001/jama.2020.8051

3. Manchanda EC, Couillard C, Sivashanker K. Inequity in Crisis Standards of Care. NEJM. Mai 13, 2020. doi: http://doi.org/10.1056/ NEJMp2011359 DOI: https://doi.org/10.1056/NEJMp2011359

4. Ministério da Saúde. Saúde define critérios de distanciamento social com base em diferentes cenários. Disponível em: https://www. saude.gov.br/noticias/agencia-saude/46666-ministerio-da-saude-define-criterios-de-distanciamento-social

5. Telessaúde RS. Qual a diferença entre distanciamento social, isolamento e quarentena? Disponível em: https://www.ufrgs.br/telessauders/ posts_coronavirus/qual-a-diferenca-de-distanciamento-social-isolamento-e-quarentena/

6. Zhang J, Litvinova, M, Liang, Y, Wang Y, Wang W, Zhao S, Wu Q, Merler S, Viboud C, Vespignani A, Ajelli M, Yu H. Changes in contact patterns shape the dynamics of the COVID-19 outbreak in China [published online ahead of print, 2020 Apr 29]. Science.2020;eabb8001. doi: http://doi.org/10.1126/science.abb8001 DOI: https://doi.org/10.1126/science.abb8001

7. Tokarski CP; Alves I. Covid-19 e violência doméstica: pandemia duplas para as mulheres. Disponível em: http://anesp.org.br/todas-asnoticias/2020/4/6/covid-19-e-violncia-domstica-pandemia-dupla-para-as-mulheres

8. Nações Unidas Brasil. Chefe da ONU alerta para aumento da violência doméstica em meio à pandemia do coronavírus. Disponível em: https://nacoesunidas.org/chefe-da-onu-alerta-para-aumento-da-violencia-domestica-em-meio-a-pandemia-do-coronavirus/

9. Centro de Estudos e Pesquisas em Emergências e Desastres em Saúde (CEPEDES/Fiocruz).Violência doméstica e familiar na COVID-19. Disponível em: https://www.fiocruzbrasilia.fiocruz.br/wp-content/uploads/2020/04/Sa\%C3\%BAde-Mental-e-Aten\%C3\%A7\%C3\%A3oPsicossocial-na-Pandemia-Covid-19-viol\%C3\%AAncia-dom\%C3\%A9stica-e-familiar-na-Covid-19.pdf

10. UNFPA Brasil. COVID-19: Um olhar para gênero. Promoção da saúde e dos direitos sexuais e reprodutivos e promoção da igualdade de gênero. Março, 2020. Disponível em: https://www.sbmfc.org.br/wp-content/uploads/2020/03/covid19_olhar_genero.pdf

11. Câmara dos Deputados dos Brasil [Internet]. Crescem denúncias de violência doméstica durante pandemia. Disponível em: www. camara.leg.br/noticias/661087-crescem-denuncias-de-violencia-domestica-durante-pandemia

12. Sociedade Brasileira de Medicina de Família e Comunidade. Recomendações da SBMFC para a APS durante a pandemia de COVID-19. $1^{\text {a }}$ Ed. Maio, 2020. Disponível em: https://www.sbmfc.org.br/wp-content/uploads/2020/05/Recomendac\%CC\%A70\%CC\%83es-daSBMFC-para-a-APS-durante-a-Pandemia-de-COVID-19.pdf

13. Camara BS, Delamou A, Diro E, Béavogui AH, Ayadi AME, Sidibé S, Grovogui FM, Takarinda KC, Bouedouno P, Sandouno SD, Okumura J, Baldé MD, Van Griensven J, Zachariah, R. Effect of the 2014/2015 Ebola outbreak on reproductive health services in a rural district of Guinea: an ecological study. Trans R Soc Trop Med Hyg. 2017;111(1):22?29. doi: http://doi.org/10.1093/trstmh/trx009 DOI: https:// doi.org/10.1093/trstmh/trx009

14. Rilay T, Sully E, Ahmed Z, Biddlecom A. Estimates of the Potential Impact of the COVID-19 Pandemic on Sexual and Reproductive Health In Low- and Middle-Income Countries. Int Perspect Sex Reprod Health. 2020;46:73?]76. Published 2020 Apr 16. doi: http://doi. org/10.1363/46e9020

15. Sochas L, Channon AA, Nam S. Counting indirect crisis-related deaths in the context of a low-resilience health system: the case of maternal and neonatal health during the Ebola epidemic in Sierra Leone. Health Policy and Planning, 32, 2017, iii32-iii39 doi: http:// doi.org/10.1093/heapol/czx108 DOI: https://doi.org/10.1093/heapol/czx108

16. Hart JT. The Inverse Care Law. The Lancet, V. 297, N. 7696, P. 405-412, 1971. DOI: https://doi.org/10.1016/S0140-6736(71)92410-X

17. Vieira PR, Garcia LP, Maciel ELN. Isolamento social e o aumento da violência doméstica: o que isso nos revela?. Rev. bras. epidemiol. [online].2020, vol.23 [citado 2020-05-21], e200033. Epub 22-Abr-2020. ISSN 1980-5497. doi: http://doi.org/10.1590/1980-549720200033 DOI: https://doi.org/10.1590/1980-549720200033

18. Roesch E, Amin A, Gupta J, García-Moreno C. Violence against women during covid-19 pandemic restrictions. BMJ 2020;369:m1712 (Published 7 May 2020). doi: http://doi.org/10.1136/bmj.m1712 PMID: 32381644 DOI: https://doi.org/10.1136/bmj.m1712

19. Marques ES, Moraes CL de, Hasselmann MH, Deslandes SF, Reichenheim ME. A violência contra mulheres, crianças e adolescentes em tempos de pandemia pela COVID-19: panorama, motivações e formas de enfrentamento. Cad. Saúde Pública [online].2020, vol.36, n.4 [citado 2020-05-21], e00074420. doi: http://doi.org/10.1590/0102-311X00074420 DOI: https://doi.org/10.1590/0102-311x00074420 
20. Lima NJSO, Pacheco LR. Violência doméstica contra a mulher na perspectiva de agentes comunitários de saúde. Rev Enferm UFPE, Recife, 10 (5): 4279-85, nov 2016. doi: http://doi.org/10.1590/S1983-14472013000100023

21. Lira CEPR, Silva PPAC, Trindade RFC. Conduta dos agentes comunitários de saúde diante de casos de violência familiar. Rev. Eletr. Enf.14(4):928-36, 2012. doi: http://doi.org/10.5216/ree.v14i4.12237 DOI: https://doi.org/10.5216/ree.v14i4.12237

22. Jacinto AMFL. Violência doméstica contra a mulher: representações e práticas do agente comunitário de saúde [dissertação]. São Paulo: Universidade de São Paulo, Faculdade de Saúde Pública;2018 [citado 2020-05-25]. doi: http://doi.org/10.11606/D.6.2018.tde15082018-154651

23. Scaranto CAA, Biazevic MGH, Michel-Crosato E. Percepção dos agentes comunitários de saúde sobre a violência doméstica contra a mulher. Psicol. cienc. prof., Brasilia , 27 (4): 694-705, Dec. 2007. doi: http://doi.org/10.1590/S1414-98932007000400010 DOI: https:// doi.org/10.1590/S1414-98932007000400010

24. Garbin CAS, Melo LMLL, Moimaz SAS, Garbin AJL, Rovida TAS. Violência intrafamiliar na rotina do agente comunitário de saúde. J Health Sci Inst.32(4):385-9, 2014. Disponível em: https://www.unip.br/presencial/comunicacao/publicacoes/ics/edicoes/2014/04_outdez/V32_n4_2014_p385a389.pdf

25. Schraiber LB, d'Oliveira, AFPL, França Junior I, Strake SS, Oliveira EA. A violência contra mulheres: demandas espontâneas e busca ativa em unidade básica de saúde. Saúde e Sociedade 9 (1/2): 3-15, 2000. doi: http://doi.org/10.1590/S0104-12902000000100002 DOI: https://doi.org/10.1590/S0104-12902000000100002

26. d'Oliveira AFPL, Schraiber LB, Hanada H, Durand J. Atenção integral à saúde de mulheres em situação de violência de gênero - uma alternativa para a atenção primária em saúde. Ciência \& Saúde Coletiva, 14(4):1037-1050, 2000. doi: http://doi.org/10.1590/S141381232009000400011

27. Ministério da Mulher, da Família e dos Direitos Humanos [Internet]. Ligue 180. Disponível em: https://www.gov.br/mdh/pt-br/naveguepor-temas/politicas-para-mulheres/ligue-180

28. Ministério da Mulher, da Família e dos Direitos Humanos [Internet]. Balanço anual: Ligue 180 recebe mais de 92 mil denúncias de violações contra mulheres. Disponível em: https:/www.gov.br/mdh/pt-br/assuntos/noticias/2019/agosto/balanco-anual-ligue-180recebe-mais-de-92-mil-denuncias-de-violacoes-contra-mulheres

29. Cidadania e assistência social [Internet]. Disque 100 vai receber denúncias relacionadas ao coronavírus. Disponível em: https://www. gov.br/pt-br/noticias/assistencia-social/2020/03/disque-100-vai-receber-denuncias-relacionadas-ao-coronavirus-1

30. IRISi.Guidance for General Practice Teams: Responding to domestic abuse during telephone and video consultations. 2020. Disponível em: https://www.lambeth.gov.uk/sites/default/files/IRIS-COVID-19-Guidance-for-GP-Teams_0.pdf

31. Home Affairs Select Committee Evidence-Respect. Respect's response to the home affairs call for evidence COVID-19 preparedness. 2020. Disponível em: https://hubble-live-assets.s3.amazonaws.com/respect/attachment/file/82/Home_Affairs_Call_for_Evidence_ Covid_19_Preparedness_April_2020_FINAL.p Secretaria de Atenção Primária à Saúde. Nota técnica 13:Recomendações acerca da atenção puerperal, alta segura e contracepção durante pandemia da COVID-19. Maio, 2020. Disponível em: http://189.28.128.100/ dab/docs/portaldab/documentos/nt_n13_2020_COSMU_CGCIVI_DAPES_SAPS_MS).pdf

32. Secretaria de Atenção Primária à Saúde. Nota técnica 10: Recomendações para as consultas ambulatoriais de saúde da mulher durante a pandemia da COVID-19. Disponível em: http://189.28.128.100/dab/docs/portaldab/documentos/ notatecnica102020COSMUCGCIVIDAPESSAPSMS.pdf

33. Secretaria de Atenção Primária à Saúde. Nota técnica 9: Recomendações para o trabalho de parto, parto e puerpério durante a pandemia COVID-19. Abril, 2020. Disponível em: http://189.28.128.100/dab/docs/portaldab/documentos/ notatecnica92020COSMUCGCIVIDAPESSAPSMS.pdf

34. Tran NT, Tappis H, Spilotros N, Krause S, Knaster S. Not a luxury: a call to maintain sexual and reproductive health in humanitarian and fragile settings during the COVID-19 pandemic. The Lancet Global Health 2020;21 Disponivel em: https://www.thelancet.com/pdfs/ journals/langlo/PIIS2214-109X(20)30190-X.pdf PMID: 32330429

35. Della Gatta AN, Rizzo R, Pilu G, Simonazzi G. Coronavirus disease 2019 during pregnancy: a systematic review of reported cases. Am J Obstet Gynecol. 2020 Apr 18:S0002-9378(20)30438-5. doi: http://doi.org/10.1016/j.ajog.2020.04.013 DOI: https://doi.org/10.1016/j. ajog.2020.04.013

36. Shanes ED, Mithal LB, Otero S, Azad HA, Miller ES, Goldstein JA. Placental Pathology in COVID-19. Am J Clin Pathol 2020;XX:0-0. doi: http://doi.org/10.1093/ajcp/aqaa089 PMID: 32441303 DOI: https://doi.org/10.1093/ajcp/aqaa089

37. Polis CB, Grimes DA, Schaffer K, Blanchard K, Glasier A, Harper C. Advance provision of emergency contraception for pregnancy prevention. Cochrane Database of Systematic Reviews 2007, Issue 2. Art. No.: CD005497. doi: http://doi.org/10.1002/14651858. CD005497.pub2 DOI: https://doi.org/10.1002/14651858.CD005497.pub2 
38. Brasil. Ministério da Saúde. Secretaria de Atenção à Saúde. Departamento de Atenção Básica. Atenção ao pré-natal de baixo risco [recurso eletrônico] / Ministério da Saúde. Secretaria de Atenção à Saúde. Departamento de Atenção Básica. - 1. ed. rev. - Brasília: Editora do Ministério da Saúde, 2013. Disponível em: http://189.28.128.100/dab/docs/portaldab/publicacoes/caderno_32.pdf

39. Dowswell T, Carroli G, Duley L, Gates S, Gülmezoglu AM, Khan-Neelofur D, Piaggio G. Alternative versus standard packages of antenatal care for low-risk pregnancy. Cochrane Database of Systematic Reviews 2015, Issue 7. Art. No.: CD000934. doi: http://doi. org/10.1002/14651858.CD000934.pub3 DOI: https://doi.org/10.1002/14651858.CD000934.pub3

40. Rocha AF. Mortalidade neonatal: assistência pré-natal em municípios do nordeste do Brasil. Dissertação de mestrado. Julho, 2013. Disponível em: https://www.arca.fiocruz.br/bitstream/icict/24321/1/412.pdf

41. Figueiredo PP, Lunardi Filho WD, Lunardi VL, Pimpão FD. Mortalidade infantil e pré-natal: contribuições da clínica à luz de Canguilhem e Foucault. Rev. Latino-Am. Enfermagem 20(1): jan.-fev. 2012. doi: http://doi.org/10.1590/S0104-11692012000100026 DOI: https://doi. org/10.1590/S0104-11692012000100026

42. Cohen MA, Powell AM, Coleman JS, Keller JM, Livingston A, Anderson JR. Special ambulatory gynecologic considerations in the era of coronavirus disease 2019 (COVID-19) and implications for future practice. Am J Obstet Gynecol. 2020 Sep;223(3):372-378. doi: 10.1016/j.ajog.2020.06.006 PMID: 32522513 DOI: https://doi.org/10.1016/j.ajog.2020.06.006

43. Engstrom E, Melo E, Giovanella L, Mendes A, Grabois V, Mendonça MHM. Recomendações para a organização da Atenção Primária à Saúde no SUS no enfrentamento da Covid-19. FioCruz. Série Linha de Cuidado COVID-19 na rede de atenção à saúde. Maio 2020.

44. ANVISA. Agência Nacional de Vigilância Sanitária. Nota técnica GVIMS/GGTES/ANVISA № 07/2020, de 08 de maio de 2020. Orientações para a prevenção da transmissão de COVID-19 dentro dos serviços de saúde. Disponível em: http://portal.anvisa.gov. br/documents/33852/271858/NOTA+T\%C3\%89CNICA+-GIMS-GGTES-ANVISA+N\%C2\%BA+07-2020/f487f506-1eba-451f-bccd06b8f1b0fed6

45. Vasconcelos EM, Vasconcelos MOD. Educação popular. In: Gusso G, Lopes JMC, Dias LC, organizadores. Tratado de medicina de família e comunidade: princípios, formação e prática. 2. ed. Porto Alegre: Artmed, 2019, p. 119. 